\title{
Information Displays and Crew Configurations for UTM Operations
}

\author{
Quang V. Dao ${ }^{1}$, Lynne Martin ${ }^{1}$, Joey Mercer ${ }^{1}$, Cynthia Wolter $^{2}$, \\ Ashley Gomez ${ }^{2}$, and Jeffrey Homola ${ }^{1}$ \\ ${ }^{1}$ NASA Ames Research Center \\ Moffett Field, CA, USA \\ ${ }^{2}$ San Jose State University Research Foundation, \\ One Washington, San Jose, CA, USA \\ \{quang.v.dao, lynne.martin, joey.mercer, cynthia.wolter, ashley.n.gomez, \\ jeffrey.r.homola\}@nasa.gov
}

\begin{abstract}
In this paper we discuss how team configuration may influence how information is shared among team members for low-altitude Unmanned Aircraft Systems (UAS) operations. NASA collected and analyzed observation data gathered during a series of field tests for the UAS Traffic Management (UTM) project. The field tests were part of a larger effort aimed at advancing the UTM concept, conducted at six test-sites spread across the USA. Ground control station (GCS) concepts, flight-crew composition, and crew-size varied within and across test-sites. Flight crews took two strategic approaches to organizing their teams. The first of the two approaches was implemented by one third of the flight crews. These crews integrated the role of UTM operator into the duties of existing crew members, merging the current roles with this new one, keeping the UTM Operator collocated with the flight crew. The remaining two thirds implemented a distributed team configuration, where a single UTM operator distributed support across multiple crews. Results from our data collection efforts revealed that UTM Operator location influenced whether flight crews used verbal communication versus displays to acquire UTM information.
\end{abstract}

Keywords: UTM $\cdot$ UAS $\cdot$ Teams $\cdot$ Situation Awareness

\section{Introduction}

The focus of this paper is on the impact of unmanned aircraft system (UAS) crew configuration on how information pertinent to a crew's objectives is used and accessed. These crews operate unmanned aircraft (UA) for diverse applications. They may fly the aircraft manually or provide high level direction if the aircraft is autonomous. We expect that most UA traffic will be autonomous because the quantity of aircraft - particularly for major commercial operations - will exceed the human operator's bandwidth for actively managing the flights. By 2035, these commercial operations will comprise more than half of the projected 250,000 concurrent UAS operations [1]. The overall UA fleet size is forecast to be 35 times the number of manned aircraft currently in operation. To meet this expected demand, NASA is currently working with academic and 
industry partners to develop a concept for a UAS Traffic Management (UTM) ecosystem [2]. The UTM system will manage traffic for unmanned aircraft that are less than $50 \mathrm{lbs}$, and only for operations occupying airspace up to $500 \mathrm{ft}$ in altitude. At the center of this ecosystem is an information exchange system that allocates active management of aircraft to automation and provides services to UAS operators for coordinating shared access to airspace [3]. In a series of four flight test campaigns NASA will observe and analyze how UAS operators incorporate UTM tasks, displays, and tools with their crews [4]. These four campaigns will be supported by four distinguishable technical capability levels (TCL).

\subsection{Flight Test Campaigns}

The TCLs build on each other and will include tests that grow in complexity. TCL 1 and 2 have already been completed. TCL 1 was limited to operations within visual line of sight and no more than 2 concurrent operations. It served to test very basic UTM functionality, which included vetting proposed flights for lateral conflicts with active operations and pre-defined constraints such as airports. In TCL 2, beyond visual line of sight (BVLOS) operations were introduced, along with enhancements such as alerting for airspace intrusion and segmented flight planning, that vetted operations for both lateral and vertical conflicts so that concurrent airspace reservations can be stacked by altitude. TCL 3 and 4 will be conducted within two years from the publishing of this paper. They will include operations over increasingly populated areas, between moderate and high UAS traffic densities, interactions between manned and unmanned operations, as well as large-scale contingency management. For a more detailed discussion of the flight campaigns and TCLs see Johnson, et al. [4], and for a more detailed discussion of NASA's concept of operation see Kopardekar, et al. [2]. The findings reported in this paper will focus on the most recent test - TCL 2.

In TCL 2, displays that provided information about the availability of mission airspace and information about where their aircraft were relative to operational boundaries, as well as other air traffic were introduced. How information was accessed depended on the configuration of the flight crew. This configuration was influenced by the role of the crew member responsible for UTM tasks and how she coordinated with the rest of the crew to disseminate UTM information for achieving mission objectives. In the next section we stage our analysis of the UTM crew with a brief discussion about teams and point to distinct features necessary for them to function.

\subsection{Share Cognition and Situation Awareness}

In this document, we define teams to be UAS ground control station crews composed of two or more individuals who have distinguishable roles, but who are interacting interdependently to achieve a common objective [5]. Endsley [6] points out 3 major features of teams from this definition. The first is that team members share common objectives. Second, team members have specific role, and, third, the members are interdependent. Commonly, functional teams will collectively form a construct called 
shared cognition [7]. Shared cognition develops when team members share four specific types of information through effective communication and coordination: (1) knowledge about a task, (2) knowledge of processes pertaining to a task, (3) knowledge about team members, and (4) their attitudes or beliefs [7]. Acquisition of the aforementioned types of knowledge allow the formation of mental models that are shared between members of a team. These models are important because individuals can then anticipate appropriate action in advance of verbal approval from others - particularly during periods of high workload.

As long as the appropriate types of information are being propagated, effective teams need only share enough information to reach a common understanding. Endsley and Jones [6] use the term shared SA to refer to information that is actually shared among team members. For UTM, this information includes an aircraft's position relative to its operational boundary and surface obstructions. The purpose of shared SA is to achieve team SA. The key difference here is that team SA is achieved when team members share an understanding that is an accurate reflection of the relevant events and system(s) in the environment. It is not always the case that shared SA leads to team SA. For example, a UA ground control station operator monitoring a display may concur with a visual observer who is also tracking an aircraft in the sky that there is enough fuel capacity to complete a mission, but both team members may neglect to identify changing wind conditions that would accelerate the burn rate on the fuel cell, effectively requiring the aircraft to return sooner than planned. In this paper, we focus on how shared SA was achieved as a function of the UAS team (i.e., flight crew) configuration, and now turn to a description of the flight test environment in which these teams operated before discussing results from our data collection efforts.

\section{$2 \quad$ Method}

A series of flight tests were conducted at six different sites located across the USA, during May and June of 2017. The campaign resulted in over 240 data collection flights. Flights not only varied in duration, but also in the environments and terrains over which they flew. The flight tests highlighted beyond visual line of sight and altitude-stratified operations. Scenarios were developed independently by each test-site to demonstrate the UTM capabilities that they had proposed, and were required to include some combination of BVLOS, altitude stratification and multiple vehicles in the air. Some test-sites created one scenario with a series of variations to capture these capabilities, while other test-sites constructed a number of unique scenarios. Test sites reviewed and modified their scenarios in one or more shakedown days, that commonly included equipment testing (e.g., testing connectivity with UTM), while data-collection days focused on completing flights meant to satisfy the test scenarios. More detailed numbers regarding how these flight activities were distributed over the six test-sites are available in Martin, et al. [8]. 


\subsection{Crew Roles and Responsibilities}

A total of 23 flight crews participated in the tests. Flight crews varied in number and affiliation: some had just two individuals, while others had approximately twelve in their crews. Flight crews from some test-sites were composed of individuals from one organization, while other test-sites' crews had members from different organizations. Primary flight crew positions included those listed in Table 1 and additional positions staffed by some, if not all, of the flight test sites are listed in Table 2.

Table 1. Crew member roles and responsibilities.

\begin{tabular}{ll}
\hline Crew Role & Crew Responsibilities \\
\hline Pilot-In-Command (PIC) & Served as the main pilot for the vehicle \\
GCS Operator (GCSO) & Worked the vehicle's flight planning and \\
& flight execution software \\
USS Operator (USS Op) & Monitored and interact with USS displays (\& \\
& NASA) \\
Launch and Software Flight Engineers & Supported specific technical aspects of the \\
& vehicle \\
Visual observers (VO) & Safety monitors who provided visual contact \\
& with the vehicles at all times \\
\hline
\end{tabular}

Table 2. Test site support personnel roles and responsibilities.

\begin{tabular}{ll}
\hline Support Role & Support Responsibilities \\
\hline UTM Manager & $\begin{array}{l}\text { Ensured the USS software was running and under- } \\
\text { took troubleshooting when needed }\end{array}$ \\
Radio control (RC) Safety Pilots & $\begin{array}{l}\text { Served as alternate pilots if the PIC needed assistance } \\
\text { Coordinated the crews and flights to conduct the test } \\
\text { Flight Test Manager }\end{array}$ \\
$\begin{array}{l}\text { scenarios properly } \\
\text { Collected observational and survey data, observers } \\
\text { NASA researchers \& Observers } \\
\text { were available to support media day and answer flight } \\
\text { team questions }\end{array}$ \\
\hline
\end{tabular}

\subsection{Crew Configuration}

Although each test-site created their own crew configurations, two types of team organization emerged with respect to UTM - integrated and distributed. In integrated teams, organization included having the USS Op role (Table 1) as a dedicated member of the flight crew, either completing USS client management tasks alone, or by having one crew member splitting the USS Op role with another role (e.g., at Test-Site 3 the flight crew consisted of two people: a GCSO/PIC/USS Op and a safety pilot/launch engineer). The advantages of having the USS Op role within a flight crew team was that this person was able to focus completely on the crew's mission and communications were reduced. The cost was the number of additional personnel, or, if the role was timeshared by one team member, that periods of high workload were compounded if all roles were busy at the same time, (e.g., at take-off and landing). 
In distributed teams, a dedicated USS Op fulfilled this role for a number of crews (e.g., at Test-Site 1, one USS Op submitted and managed the flight volumes for four flight crews, where each flight crew consisted of a PIC, a GCSO, and a launch engineer). Three test-sites took this "hub-\&-spoke" approach - Test-Sites 1, 4 and 5. The advantage of separating out the USS Op role was that this person became a specialist and overall required manpower was reduced. The cost was the increase in communications load, as the USS Op had to stay in contact with all the flight crews s/he was serving, and the workload related to managing multiple flights in the case that one flight crew/vehicle was having an off-nominal event.

\subsection{Tools and Displays}

Equipment available at each Ground Control Station (GCS) location varied widely across (and sometimes within) test-sites. At most GCSs, several displays were available to the flight crews to give them information about their vehicle's flight, and some also included displays to show surrounding operations, and/or aspects of the UTM system. For example, Test-Site 3 provided four screens for its GCSO/PIC/USS Op. These individuals were not in line of sight (LOS) of their vehicle. Standard tools shown on their displays were flight planning/execution software, a USS client, and a fusion of radar, multi-lateration systems and GCS telemetry. The fourth screen was available to show other information of the GCSO/PIC/USS Op's choice, including weather, vehicle and USS data, radio frequency usage, etc. Other test-sites, which had more mobile/portable GCSs, used fewer displays. At Test-Site 5, for example, flight crews had a handheld controller, and one display showing the autopilot software for their vehicle. These flight crews did not have access to a display of UTM information. Instead, UTM information was verbally relayed to them by radio from a centralized location, where the USS Op had such a display.

All test-sites used at least one surveillance system to provide information about the airspace not provided by vehicles' on-board sensors (GPS, ADS-B), helping to identify other manned and unmanned aircraft flying near the test-site. A NASA-built iOS application called insight UTM (iUTM), provided visualizations of UTM system information and current operations, and was made available to the test-sites. Test-Sites 3 and 5 elected to use iUTM as an additional situation awareness display. To participate in these tests, participants needed to have certain basic capabilities, but the manner and extent by which the partners met those requirements differed. These differences are not examined in this paper.

\subsection{Procedure}

During test days, teams of two researchers collected data from the participants at each test-site about their experiences. To the extent possible, researchers observed all flight crews at some point across the test days. Data were collected in a number of ways:

- observations of participants during flights,

- questionnaires administered at the end of the day, 
- interviews conducted at the end of the day.

All of these methods solicited feedback on how flight crews used information and where they looked for it. In total, 18 end-of-day group interviews were collected across the six test sites, totaling nearly nine hours of recording. Survey items were presented to the participants in the context of the research objectives of the test-site's test scenarios. Approximately 40 questions were generated in the flight test survey, but conditions were set so that participants only answered around 15 at any one time. There were only three or four questions in each shakedown survey. Most questions used a seven-point rating format, but some were multiple choice or open-ended.

\section{$3 \quad$ Results}

Recurring themes that surfaced from field observations convey how information was shared as a function of crew configuration.

\subsection{Sources of Information}

As the USS interface provides an additional set of information to the crew, team members were asked both before and during flight tests about the source of information they used. Between a shakedown questionnaire and an end of day survey, participants were asked five questions about where they looked during flights to gain information about their vehicle. Numbers of responses varied from 24 to 45 to the end of day survey and were 19 for the shakedown survey.

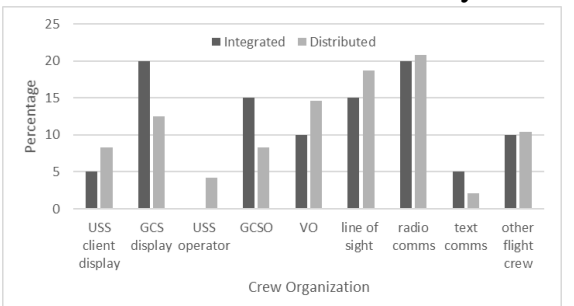

Fig. 1. Percentage of reported usage of information sources by crew configuration before flight test participation.

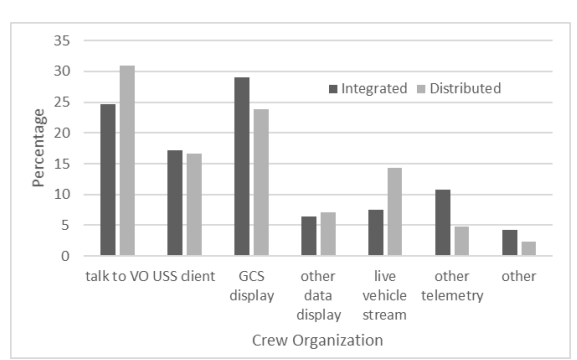

Fig. 2. Percentage of reported usage of information sources by crew configuration after flight test participation.

Before taking part in the flight test, respondents estimated that they would look to other personnel (e.g., the GCSO or the USS Op) three quarters of the time (Figure 1) and look at displays about $25 \%$ of the time to gather information about their own vehicle, with talking via radio being the most frequently chosen option $(n=14)^{1}$. Most respondents reported seeking vehicle information from more than one source. Both integrated and distributed crews report primarily using radio comms (around 20\% of the

\footnotetext{
${ }^{1}$ Note that the question options were uneven, as six personnel roles were listed but only three displays.
} 
time) and line of sight (17\%) to gain information about their vehicle. They did report using tools (e.g., USS client) to gather information, but to a lesser extent than relying on reports from other people. Their responses were very similar for where they would gather information about other vehicles flying in the area. Before taking part in the flight test, respondents estimated that they would look to other personnel for information $63 \%$ of the time and look at displays about $19 \%$ of the time, with talking via radio, again, being the most frequently chosen option $(n=14)^{2}$.

After days when they had flown BVLOS missions, participants were asked a question similar to that above. Most respondents, again, reported looking at more than one source of information to keep track of their vehicle when it was BVLOS. Three sources of information stood out as most frequently used. About $27 \%$ of the time, respondents looked at their ground station display and talked to their $\mathrm{VO}$ to gather vehicle position information (Figure 2). The USS client display was the third most popular source of information, chosen 23 out of 135 times $(17 \%)$. Integrated crews reported going to a tool most often to find information about their vehicle, while distributed crews reported they primarily talked to their $\mathrm{VO}$ to gain information.

There are two encouraging aspects to the differences between participants' answers before the flight tests and after. The first is that usage of the USS client increased, between shakedowns and post-flight data collection. The percentage of the time participants said they had looked at USS client information more than doubled from just over $7 \%$ to just over $17 \%$. The second, is a focusing of where participants looked for information (which is not readily shown in Figures 1 and 2) from an average of just under four sources (3.8) to fewer than three (2.5).

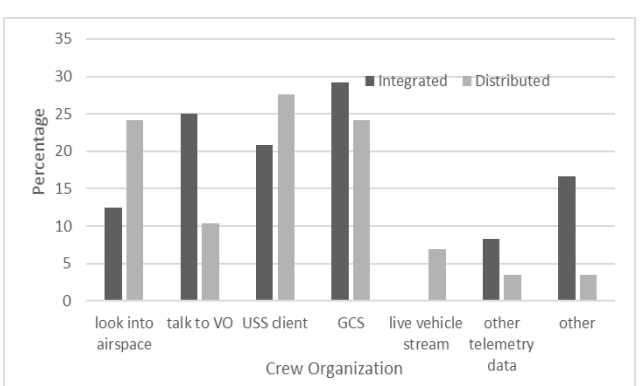

Fig. 3. Percentage of reported source usage to obtain information about altitude stratified flights, by crew configuration.
Participants were asked the same question again in the context of acquiring information for altitude-stratified flights. Again, most respondents reported looking at more than one source of information to keep track of relative aircraft positions when their flight was altitude-stratified with another. On average, participants reported looking at three sources of information. Integrated crews looked at their GCS display, the USS client display and talked to their VO most often (Figure 3). Distributed crews looked at their USS client display, their GCS, and into the airspace. In Figure 3, the slight difference between crews of different organizations, seen in Figure 2, is more marked, with distributed crews shifting to look at their tools a little more. However, this could be a result of the conditions at the time or the exact nature of the flight.

\footnotetext{
${ }^{2}$ Note that the question options were uneven, as six personnel roles were listed but only two displays.
} 


\subsection{Finding and Using Information}

When asked questions about the process of finding information rather than the source of data, half of the respondents (16 of 32) reported they were "easily" able to find all the information they needed to support their decisions ${ }^{3}$. Integrated crews seemed to find the information they needed more easily, with two thirds reporting they could "easily find all the information they needed" as opposed to only forty percent of distributed crew personnel.

Ease of finding information plays into how hard crews have to work to achieve their tasks. On average, participants reported their workload as "medium" ( $\mathrm{m}=4.16$ out of $7)$, with participants from integrated crews feeling they were more loaded $(\mathrm{m}=4.8)$ than participants from distributed crews $(m=3.8)$ (Figure 4$)$. It is possible that integrated crews would have a higher load at busier times because the USS Operator was often not an additional person in the team. The USS Op roles had been integrated with other team roles, and were either allocated across the group or were an additional set of tasks for one team member. For distributed crews, the USS Op role was filled by an additional person, who was not located with the team. Under nominal conditions, the crew would not be aware of any additional UTM activity. However, for this series of flight tests, the case where multiple vehicles simultaneously had off nominal events was not tested, and it is hypothesized that distributed crews may experience higher workload or a lower awareness of local operations under these conditions.

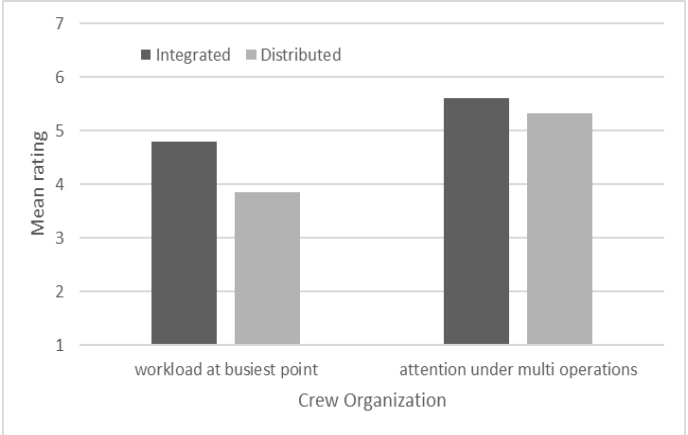

Fig. 3. Mean level of workload and level of attention, shown by crew organization.
Participants were also asked to rate how their level of attention changed when there were multiple vehicles flying. Integrated crew members reported their level of attention changed and they "increased their vigilance" ( $\mathrm{m}=5.8$ out of 7 ) (Fig. 4). Distributed crew members also increased their vigilance "a little" $(\mathrm{m}=5.3)$, but not as much as integrated crew-members. When there were multivehicle operations, and some of these vehicles were out of the line of sight for the team on the ground, distributed team members may have had less awareness for these other vehicles if they did not make an effort to check in with their USS Op. Respondents often said that they gathered information from other people rather than displays, e.g., "all information was provided by my eyes, crew and OC radio calls" (Test-Site 2 participant). Initially, crews intended to look for the potential locations of other vehicles during their flight planning, whereas, while their vehicle was airborne, they intended to only seek out information about other flights if they could see and/or hear this other vehicle in the vicinity. However, participants reported

\footnotetext{
${ }^{3} 9 \%$ of respondents reported they had no display, however, absence of a visual display does not mean that the participant received no information, as many flight crews were designed to receive information via voice.
} 
they, in fact, took a slightly different approach, where close to $30 \%$ of the time they had looked for other vehicles on their displays "all the time". Integrated crews reported using their displays at some level $75 \%$ of the time and distributed crews reported using their displays at some level $38 \%$ of the time to gain information about other vehicles. This affected their use of their USS client, a third of the time participants reported looking at the USS displays themselves, and the other two thirds of the time they asked the USS Operator to report the USS client information to them. It should be noted that this depended on a participant's role in the flight crew - whether they were reporting or being reported to. However, discussions highlighted that additional verbal communications were required when the UTM Op was remote from the rest of the crew. A small difference between integrated and distributed crews, that illustrates how the team workflow was different between the two crew organizations, was that $20 \%$ of the time, distributed crews reported only gaining USS client information when there was an alert, whereas integrated crews reported that they "always during concurrent operations," or "always," looked at their USS client. This reflects the greater availability of UTM information in integrated crews.

In debriefs, operators were also asked about information that they would want to gain from their displays/ tools and their team about their own flight and others. From the debrief transcriptions (see [8] for more information). Crews noted they wanted to be able to see immediately all aspects of their vehicle health, performance, and location. They also wanted to be able to find out location and health information about other flights in their vicinity. They were interested in receiving alerts about issues with their own vehicles and with others, and some suggested that they wanted their USS to suggest courses of action, give an account of why issues arise, or how crews might recover from a situation. However, this is a substantial quantity of information, and crews noted occasions during the flight tests when they experienced both visual and aural clutter from their displays. There were concerns that too much data was available and that crews could not pay attention to all of it without being distracted. Although the amount of information that a crew was able to attend to depended to some extent on the size of the team, the debrief and observation comments suggest that information needs to be carefully prioritized and then layered within tools to ensure that the most pertinent information is the most readily available, but all information can be obtained if needed.

Crews were keen to share as much information as possible with support personnel, or "home base," suggesting streaming raw data from their vehicle to these locations. They noted that consistency/standardization of information and formatting on a USS GUI (graphical user interface) is also needed for these remote personnel (including the USS Operator when teams are distributed and $\mathrm{s} / \mathrm{he}$ is managing a number of flights from a distant location) to be able to compare across, and understand multiple flights. The information that operators felt they should broadcast to others concerned off nominal vehicle states rather than nominal data. Operators stated that a USS needs to report out, off-nominal events occurring with a user's own operation, such as non-conforming or rogue states, lost links, return-to-base (RTB), and RTB procedures or intent. However, interviewees emphasized that the community needs to agree on the terminology for each of the states that is broadcast. Another point crews made about broadcasting off nominal states is that the crews themselves cannot be required to send these messages, they will be too busy managing the event. This suggests that off nominal information broadcasts through USS clients will need to be automatically triggered. 


\section{Discussion}

In this paper we reported on data collection efforts from UTM flight tests. Observation data, surveys, and interviews point to at least an informal conclusion that crew configuration guides how UAS flight crews share information. Principle devices for information acquisition about UTM events came from either displays or requested verbally from a UTM operator. Overall, when crews were integrated, they turned to displays for UTM information, while distributed groups preferred verbal communication. Mean rated workload for integrated crews were higher than for distributed crews. This difference may have been due to integrated crews having to burden a single individual with both UTM responsibilities and existing ground station activities. In contrast, the UTM operator was a standalone individual in distributed crews, who did not have a dual role.

To facilitate safe and efficient operations, flight crews needed fast access to easily understandable information about the current mission, nearby operations, and the surrounding environment. As noted above, some feedback suggested it was not a lack of information but that crews sometimes struggled to extract the information they needed from the displays they used in the flight tests - information was buried too deeply in the tool given the time available and other activities occurring, or messages were difficult to interpret. Ease of information access will likely have the most impact on shared SA for integrated crews, based on our results.

Some information participants said they just did not need, although opinion was divided on this. When the information displayed was perceived as unreliable, its usefulness was diminished as operators lost trust and sought out alternate information sources. For example, observers at different sites noted instances of multiple sources for position data showing conflicting information at the same time. Crews considered not just what information they would like to receive but also how it is presented. Many teams liked audio presentation of messages, emphasizing simple wording and that audio presentation should be used selectively. They also noted that the environmental conditions in the field sometimes make visual displays challenging to use. The mode in which information is propagated will likely play the largest role in shared SA for distributed teams. In lieu of the UTM Operator being present, verbal communication might have a positive effect on trust and reliance of the information shared - particularly where verbal communication suggests that the shared information has been vetted by another human.

Acknowledgments. The authors would like to thank our partners from academia and industry for their participation in the UTM project.

\section{$5 \quad$ References}

[1] "Unmanned Aircraft Systems (UAS) Service Demand 2015-2035 Literature Review \& Projections of Future Usage,” Cambridge, MA, 2013.

[2] P. Kopardekar, J. Rios, T. Prevot, M. Johnson, J. Jung, and J. E. I. Robinson, "Unmanned aircraft system traffic management (utm) concept of operations," Am. Inst. Aeronaut. Astronaut., 2016. 
[3] J. Rios et al., "UTM Data Workign Group Demonstration 1 Final Report," Moffett Field, CA, 2017.

[4] M. Johnson et al., "Flight Test Evaluation of a Traffic Management Concept for Unmanned Aircraft Systems in a Rural Environment," in Twelfth USA/Europe Air Traffic Management Research and Development Seminar (ATM2017), 2017.

[5] E. Salas, T. L. Dickinson, S. Converse, and S. I. Tannenbaum, "Toward an understanding of team performance and training," in Teams: Their training and performance, R. W. Swezey and E. Salas, Eds. Norwood, NJ: Ablex, 1992, pp. 3-29.

[6] M. R. Endsley and D. G. Jones, "Designing to Support SA for Multiple and Distributed Operators," in Designing for Situation Awareness, 2nd ed., New York, NY, USA: Taylor \& Francis Group, 2012,pp. 193-218.

[7] K. A. Wilson, J. W. Guthrie, E. Salas, and W. R. Howse, "Team Process," in Handbook of Aviation Human Factors, J. A. Wise, D. V. Hopkin, and D. J. Garland, Eds. Taylor \& Francis Group, 2010, pp. 9-1-9-17.

[8] Martin, L., Gomez, A., Wolter, C., Mercer, J., Homola, J. \& Dao, A.Q. (in press). TCL2 National Campaign Human Factors Brief - version 1.1, NASA Technical Manual, NASA Ames Research Center, CA. 